\title{
Survey on Knowledge and Attitudes on Vitamin D and Sunlight Exposure in an Urban Population in Vietnam
}

\author{
Lan T. Ho-Pham ${ }^{1,2}$ and Mai T. T. Nguyen ${ }^{1}$ \\ ${ }^{1}$ Department of Internal Medicine, Pham Ngoc Thach University of Medicine, Ho Chi Minh City, Vietnam \\ 2Department of Rheumatology, People's Hospital 115, Ho Chi Minh City, Vietnam
}

\begin{abstract}
Objective. Vitamin D deficiency has been shown to be a risk factor for various chronic diseases and mortality. It is not clear why vitamin D deficiency affects a large proportion of individuals living in tropical Asian countries. The present study sought to examine the knowledge, behavior and attitudes of Vietnamese men and women toward vitamin D and sunlight exposure.
\end{abstract}

Methodology. The study was designed as a cross-sectional survey of a population in Ho Chi Minh City, Vietnam. Study participants were randomly drawn from the general community, supermarkets and hospital visitors. Each participant was given a questionnaire relating to knowledge on vitamin $D$ and behavior toward sunlight exposure. The questionnaire was previously tested for reliability and accuracy in 20 participants.

Results. A total of 1,536 individuals aged 14 to 85 years participated in the study. Fifty three percent of participants did not like being exposed to sunlight. On the average, most reported approximately 14 hours per week under the sun. Majority $(81 \%)$ reported that they had heard of vitamin D from newspapers $(32 \%)$, friends $(20 \%)$ or radio and television $(13 \%)$. However, their knowledge about the source of vitamin D was inadequate: $37 \%$ thought that vitamin D comes from the sun, $28 \%$ from foodstuff and the sun, while $17 \%$ did not know the source of vitamin D. Analysis of the determinants of knowledge of vitamin $\mathrm{D}$ suggested that only educational level was a significant predictor of vitamin $\mathrm{D}$ knowledge.

Conclusion. A negative attitude toward sunlight exposure and inadequacy of knowledge on vitamin D could adversely affect bone and general health in the community. The data also provides a basis for developing public health strategies for the prevention of vitamin D deficiency in the Vietnamese population.

Keywords: vitamin D; osteoporosis; knowledge; behavior; attitude

\section{INTRODUCTION}

Vitamin D plays an important role in the regulation of calcium and bone metabolism. ${ }^{1}$ Vitamin D deficiency has been shown to be associated with-but not proven to be causally related to-an increased risk of diabetes, cancer, cardiovascular diseases, tuberculosis and even fever. ${ }^{2-12} \mathrm{~A}$ recent meta-analysis showed that vitamin $\mathrm{D}$ deficiency is associated with an increased risk of all-cause mortality. ${ }^{13-16}$ Maintaining an adequate level of vitamin D may reduce the burden of chronic diseases and contribute to better health outcomes.

In Asia, even in tropical countries, the prevalence of vitamin D deficiency has been estimated between 35\% to $57 \% .17,18$ The reason for vitamin D deficiency in people living in tropical countries remains unclear. In Vietnam and in other Asian countries, women prefer to have fair skin. Light-colored skin is perceived as a symbol of elite status, in contrast to dark skin which is usually associated with a lower socio-economic class. Moreover, among Asian women, pale or fair skin is believed to help improve appearance and enhance job prospects. Asian women tend to avoid the sun, which they believe will darken their skin color and cause wrinkles. Vietnamese women, particularly the younger ones, routinely cover their bodies when going outdoors, in the belief that this could prevent sunburn. Such behavior may contribute to vitamin D deficiency in the general population.

Ho Chi Minh City has a rapidly expanding young population. The city has a population of 6.4 million with a density of 7,943 individuals per square mile. The current annual average gross domestic product (GDP) of the city was USD 2,180 (adjusted GDP for purchasing power parity was USD 10,870). ${ }^{19}$
ISSN 0857-1074

Printed in the Philippines

Copyright (C) 2012 by the JAFES

Received February 8, 2012. Accepted April 11, 2012.
Corresponding author: Lan T. Ho-Pham, MD

Department of Internal Medicine

Pham Ngoc Thach University of Medicine

520 Thanh Thai Street, District 10

Ho Chi Minh City, Vietnam

Tel. No.: 865-2435

Fax No: 865-0025

E-mail: thuclanhopham@pnt.edu.vn 
Knowledge, attitude and behavior play an important role in the prevention and control of diseases. Therefore, the primary aim of this study is to assess the knowledge, behavior and attitude toward vitamin $\mathrm{D}$ and sunlight exposure of a group of Vietnamese individuals in Ho Chi Minh City. The secondary aim of the study is to identify patterns and determinants of knowledge and behavior.

\section{METHODS}

\section{Study design and procedure}

The study was a cross-sectional survey conducted in Ho Chi Minh City, Vietnam in January 2009.

Three teams of interviewers were sent to supermarkets and bus stations to interview participants. Shoppers and passengers were randomly approached and invited to answer a questionnaire concerning vitamin $\mathrm{D}$ and sunlight. In addition, relatives visiting patients in a major tertiary hospital in Ho Chi Minh City were also enjoined to participate in the study. The study protocol and all procedures were approved by the university's Research Committee. As per the committee's recommendation, written informed consent was not sought prior to study participation.

Participants completed a single questionnaire with assistance from a trained interviewer. The interview process was approximately 5 minutes. Participants were informed that their responses were anonymous and participation was entirely voluntary. All potential participants approached agreed to take part in the study. No names and address details were recorded to ensure anonymity.

\section{Questionnaire}

The instrument used in this study was a validated questionnaire modeled according to the questionnaire used by Kung et al., in Hong Kong ${ }^{20}$ translated into Vietnamese. It is divided into 4 main sections: demographic details, knowledge, behavior and attitudes

We conducted a test-retest reliability study of the questionnaire in a sample of 20 participants, with an intervening period of two weeks. The coefficient of reliability ranged between 0.75 and 0.95 for the 16 questions. There was no significant difference in the reliability coefficients between men and women. Analysis of reliability by age group was not conducted because of the limited sample size.

\section{Data analysis}

Descriptive statistics were used to characterize the sample. Frequency tables were constructed per question, and were subsequently stratified according to demographic characteristics. Absolute and relative frequency of response for each question was first obtained for the whole sample for subgroups stratified by demographic characteristics. Bivariate relationships between variables were examined using t-tests and Chi squared tests for numerical and categorical variables, respectively. To assess the independent effects of demographic characteristics, knowledge and belief on behavior, the logbinomial regression model was performed for each behavior. All analyses were performed using the Rstatistical software. ${ }^{21}$

\section{RESULTS}

\section{Sample characteristics}

A total of 1536 participants between 18 and 85 years of age were surveyed (Table 1). The average age of the participants was $34 \pm 14$ years (mean $\pm \mathrm{SD}$ ). Majority of participants were female $(58.3 \%)$, students $(41.5 \%)$, with secondary education $(71.1 \%)$, and worked indoors (85.4\%).

\begin{tabular}{|c|c|c|}
\hline Characteristics & Number & Percentage \\
\hline \multicolumn{3}{|l|}{ Gender } \\
\hline Male & 641 & 41.7 \\
\hline Female & 895 & 58.3 \\
\hline \multicolumn{3}{|l|}{ Age group (year) } \\
\hline$<21$ & 230 & 15.0 \\
\hline $21-30$ & 588 & 38.3 \\
\hline $31-40$ & 237 & 15.4 \\
\hline $41-50$ & 257 & 16.7 \\
\hline $51-60$ & 162 & 10.6 \\
\hline$>60$ & 62 & 4.0 \\
\hline \multicolumn{3}{|l|}{ Occupation } \\
\hline Student & 638 & 41.5 \\
\hline Salesperson & 212 & 13.8 \\
\hline Laborer & 86 & 5.6 \\
\hline Office worker & 305 & 19.9 \\
\hline Professional & 41 & 2.7 \\
\hline Retirees & 75 & 4.9 \\
\hline Housewife & 143 & 9.3 \\
\hline \multicolumn{3}{|l|}{ Education } \\
\hline Primary & 91 & 5.9 \\
\hline Secondary & 1087 & 71.1 \\
\hline College & 106 & 6.9 \\
\hline University & 246 & 16.1 \\
\hline \multicolumn{3}{|l|}{ Working condition } \\
\hline Indoor & 1311 & 85.4 \\
\hline Outdoor & 224 & 14.6 \\
\hline
\end{tabular}

\section{Attitude and behavior toward sunlight}

Participants mostly responded that they liked going out in the sun or liked to be exposed to the sun $(47 \%, \mathrm{n}=722)$ (Table 2). More men, compared to women, liked going out in the sun (53\% and $43 \%$, respectively; $p=0.001)$. Elderly men and women responded more positively about sun exposure compared with younger individuals $(76 \%$ and $40 \%$, respectively). Retirees were more likely to expose themselves to sunlight (76\%), in contrast to laborers (57\%), students (39\%), and salespersons (48\%).

On average, the participants spent approximately 14 hours per week exposed to the sun. Around $77 \%$ of women and $17.4 \%$ of men reported that they had adequate exposure to sunlight. Approximately $60 \%$ of participants reported that 
they often used parasols, and $15 \%$ often used sunscreen products with sun protection factor (SPF) greater than 15 .

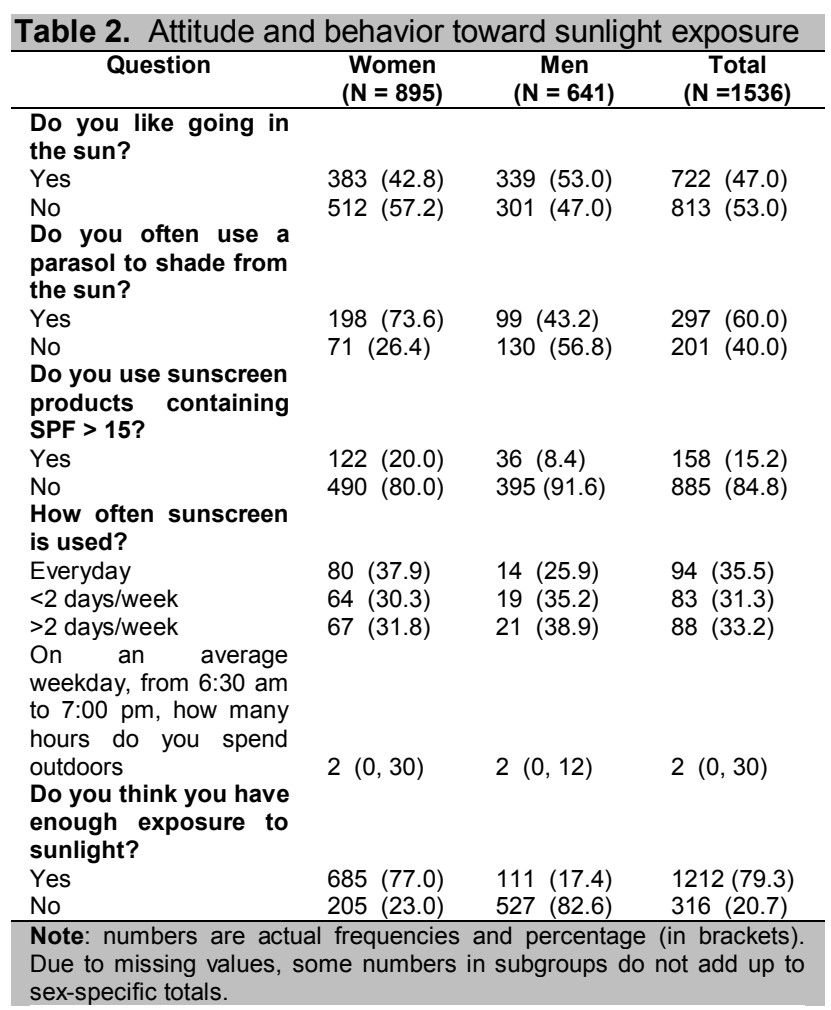

\section{Knowledge of vitamin D}

A significant majority of women (80\%) and men $(81.4 \%)$ reported that they had heard of vitamin D. Usual sources of information were newspapers $(32 \%)$, friends $(20 \%)$, radio and television $(13 \%)$, and other sources, including the internet. However, knowledge about the source of vitamin D was not adequate: $37 \%$ thought that vitamin D comes from the sun, another $28 \%$ thought that the sources of vitamin $\mathrm{D}$ are foodstuff and the sun, and $17 \%$ did not know the source of vitamin $\mathrm{D}$ (Table 3).

When asked about the benefits of vitamin $\mathrm{D}$, majority of participants thought that vitamin $\mathrm{D}$ had beneficial effects on general health $(80 \%)$, particularly bone health $(88 \%)$. Analysis of determinants of knowledge of vitamin D suggested that participants of younger age and higher education levels had better knowledge of vitamin D compared to those in the older age groups or lower levels of education.

About $70 \%$ of participants responded that they were prepared to undergo testing to know their vitamin D levels. They were also prepared to take vitamin D supplementation if found deficient $(85 \%)$.

\section{Predictors of knowledge and behaviors}

The probability of being enthusiastic about exposure to the sun was independently related to gender, age, educational level and working environment (Table 4). Compared with males, females were less likely to respond to like going in the sun [17\%, 95\% CI (9 to 25\%)]. Furthermore, younger age, having secondary or tertiary education, and working indoors were associated with being less likely to be exposed to sunlight. Knowledge on vitamin D (particularly benefits on bone health) was significantly related to higher educational level.

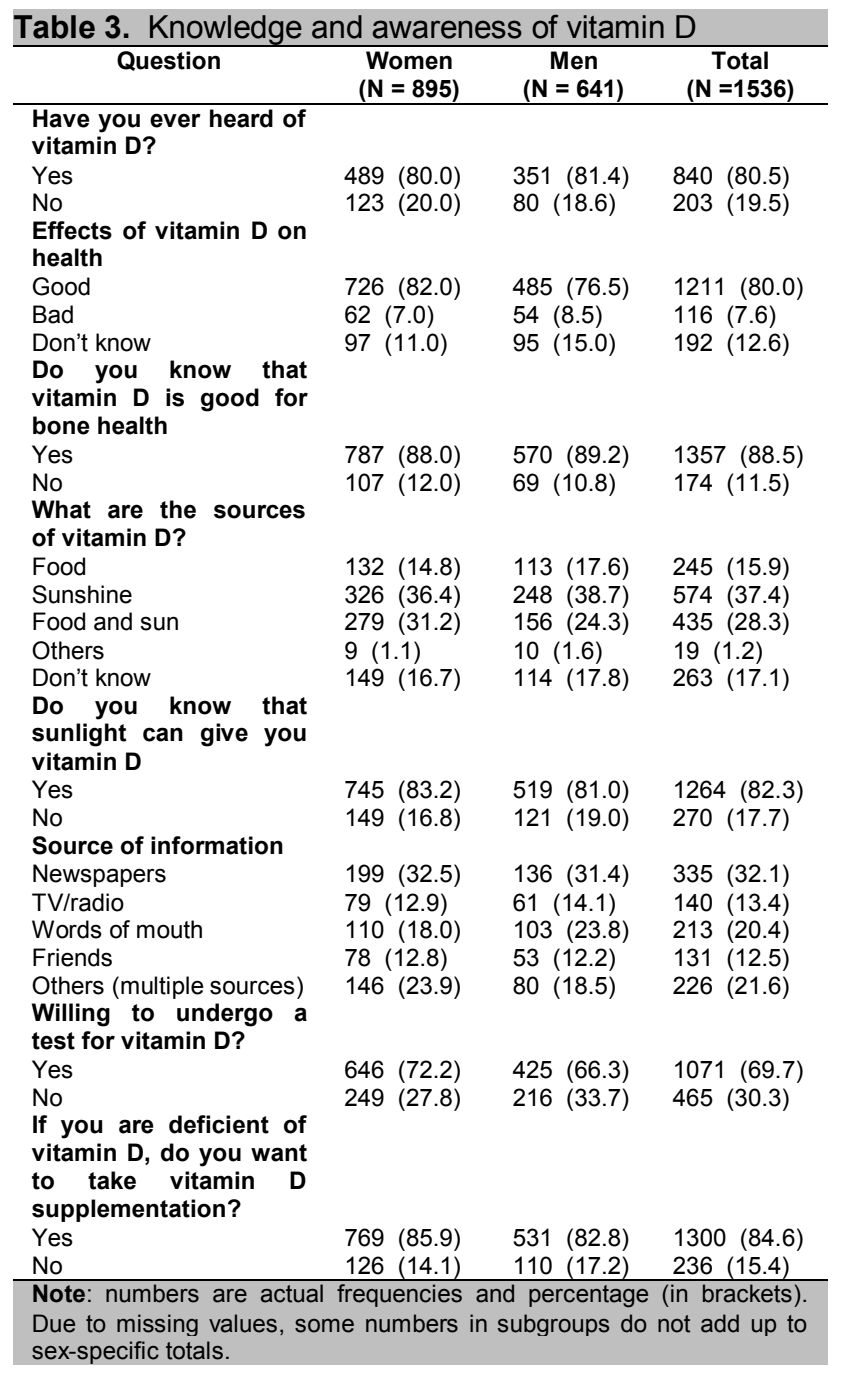

\section{DISCUSSION}

The classical function of vitamin D is to regulate calcium absorption and excretion. ${ }^{22,23}$ Thus, maintaining adequate vitamin $\mathrm{D}$ and calcium is recognized as an important means of prevention of osteoporosis and fragility fractures in the general population. The success of health promotion and community-based prevention of osteoporosis depends in part on the knowledge and behavior of members within the community. In this study, we found that the knowledge of vitamin D in an urban population in Vietnam is inadequate, and almost half of the population did not like to be exposed to the sun. This finding is consistent with the reality that many Asians, despite living in tropical regions, have vitamin D insufficiency or deficiency. ${ }^{24-26}$ Our findings also suggest that a large proportion of the Vietnamese population may have vitamin D insufficiency. 
Table 4. Factors associated with being like to contact with the sun, and recognition that vitamin $D$ is beneficial to health or bone health

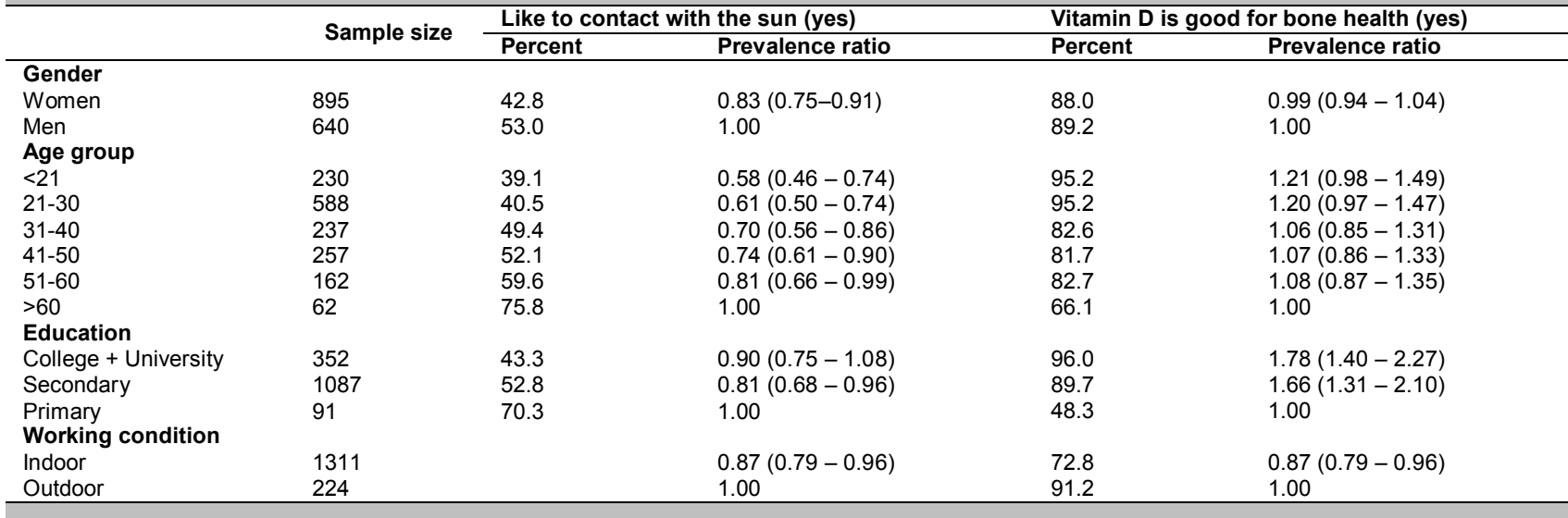

\section{Survey questions}

What is your age?

What is your occupation?

Do you work mainly indoors or outdoors?

Do you like to have contact with the sun

Do you often use a parasol to shade from the sun?

Do you use sunscreen products containing SPF $>15 ?$

On an average weekday, from 6:30 am to 7:00 pm, how many hours do you spend indoors?

Do you think you have enough exposure to sunlight

Have you ever heard of vitamin D?

What does vitamin $\mathrm{D}$ do?

What are the sources of vitamin D?

Do you know that vitamin $D$ is good for bone health?

Do you know that sunlight can give you vitamin D?

Are you willing to undergo a vitamin $\mathrm{D}$ test?

If you are deficient of vitamin D, do you want to take vitamin $D$

supplementation?

Figure 1. Survey questions

The present findings are highly consistent with a previous survey in Hong Kong. ${ }^{20}$ In their study, approximately $62 \%$ participants responded that they did not like going out in the sun-similar to our findings where $57 \%$ of women had the same response. In our study, $80 \%$ of women did not use any sunscreen product, comparable to the Hong Kong findings (81\%). In terms of knowledge, they reported that $73 \%$ of participants were aware of vitamin D, which was equivalent to our results (80\%) after accounting for sampling variation. However, while $37 \%$ of our participants thought that the sun was the main source of vitamin D, only $23 \%$ responded similarly in Hong Kong. Taken together, these data show that the two Asian populations share very similar attitude, behavior, and knowledge of vitamin D and sunlight exposure.

There were some demographic variations in vitamin D knowledge and behavior toward sunlight exposure in this study. These variations are largely consistent with the published literature. As noted in a previous study, vitamin $\mathrm{D}$ knowledge, female gender and having attended higher education were associated with less enthusiasm to be exposed to the sun. ${ }^{20}$ These findings suggests that doctors and health professionals should be particularly careful in providing dietary advice to individuals with these demographic characteristics. They are more likely to need detailed information in order to implement any lifestyle changes.

We observed a lack of consistency between attitude toward sunlight exposure and knowledge of vitamin D. While individuals working mainly indoors responded that they did not like to be exposed to the sun, the same individuals also recognized that vitamin D was good for health and bone health. Further analysis identified that these individuals were mainly university students and office workers.

The present findings should be interpreted within the context of the study's strengths and limitations. Because the study was based on a large sample size, and the participants were drawn from various occupational and educational backgrounds, these results have a high degree of external validity and generalizability. The participation was entirely voluntary, and direct interview was conducted without any drop out. However, the limitations of this study include the fact that the participants were slightly younger than the general population, which limit the generalizability of the results to the total population. Because the study was conducted in the Vietnamese population, whose lifestyle and cultural background may be different from other populations, the present findings may not be consistent with those in other parts of the world.

Despite these limitations, our findings have important implications on the prevention of vitamin D deficiency in the general population in Vietnam. The understanding of attitudes, behaviors and knowledge of the community may help design more appropriate public health programs. Our findings suggest that educational intervention programs may be targeted to reach younger women with high levels of education, especially those who have negative perceptions on sunlight exposure. Since the sources of information were mainly newspapers, television and radio, mass media campaigns with national coverage may be more preferable than educational materials or talks in community organizations. 
In recent years, there has been increasing interest in the influence of vitamin D on tissues other than bone. ${ }^{27,28}$ It is also recognized that living in tropical areas does not necessarily protect against vitamin D deficiency. The present study findings help explain why there was a high prevalence of vitamin D deficiency in Asian populations, particularly among young women.

In summary, the present data showed that approximately half of the sampled urban population in Vietnam had negative attitudes toward sunlight exposure, despite their recognition that vitamin $\mathrm{D}$ has beneficial effects on health. Given the importance of vitamin D in maintaining good health, these results imply that there is a need to develop effective strategies to educate the general community about the prevention of vitamin $\mathrm{D}$ deficiency.

\section{Acknowledgements}

The authors would like to thank Tran Quoc Dat, Mai Trong Linh, Nguyen Thi Thanh Thao, Vo Thi Thuy An, Nguyen Hai Dang and Pham Ngoc Khanh for their assistance in the recruitment and interview of participants. We thank Prof. Tuan V. Nguyen of the Garvan Institute of Medical Research for helping design the study and editing the manuscript.

\section{References}

1. Holick MF. Evolution and function of vitamin D. Recent Results Cancer Res 2003;164:3-28.

2. Pittas AG, Dawson-Hughes B, Li T et al. Vitamin D and calcium intake in relation to type 2 diabetes in women. Diabetes Care 2006;29(3):650-6.

3. Carbone LD, Rosenberg EW, Tolley EA et al. 25-hydroxyvitamin D, cholesterol, and ultraviolet irradiation. Metabolism 2008;57(6):741-8.

4. Holick MF. Calcium plus vitamin D and the risk of colorectal cancer. N Engl J Med 2006;354(21):2287-8.

5. Wang TJ, Pencina MJ, Booth SL et al. Vitamin D deficiency and risk of cardiovascular disease. Circulation 2008;117(4):503-11.

6. Wilkinson RJ, Lange C. Vitamin D and tuberculosis: New light on a potent biologic therapy? Am J Respir Crit Care Med 2009;179(9):7402.

7. Amento EP. Vitamin D and the immune system. Steroids 1987;49(13):55-72.

8. Hayes CE, Nashold FE, Spach KM et al. The immunological functions of the vitamin D endocrine system. Cell Mol Biol (Noisy-le-grand) 2003;49(2):277-300.

9. Hayes J, Dave S, Rogers C et al. A national survey in England of the routine examination of the newborn baby. Midwifery 2003;19(4):27784.

10. Lieberman D, Lieberman D, Friger MD. Seasonal variation in hospital admissions for community-acquired pneumonia: A 5-year study. J Infect 1999;39(2):134-40.

11. Shek LP, Lee BW. Epidemiology and seasonality of respiratory tract virus infections in the tropics. Paediatr Respir Rev 2003:4(2):105-11.

12. Thompson WW, Shay DK, Weintraub E et al. Influenza-associated hospitalizations in the United States. JAMA 2004;292(11):1333-40.

13. Autier P, Gandini S. Vitamin D supplementation and total mortality: A meta-analysis of randomized controlled trials. Arch Intern Med 2007;167(16):1730-7.

14. Melamed ML, Michos ED, Post W et al. 25-hydroxyvitamin D levels and the risk of mortality in the general population. Arch Intern Med 2008;168(15):1629-37.

15. Pilz S, Dobnig H, Tomaschitz A et al. Vitamin D and cancer mortality. Cancer Epidemiol Biomarkers Prev 2009;18(1):359.

16. Pilz S, März W, Wellnitz B et al. Association of vitamin D deficiency with heart failure and sudden cardiac death in a large cross-sectional study of patients referred for coronary angiography. J Clin Endocrinol Metab 2008;93(10):3927-35.

17. Holick MF. Vitamin D deficiency. N Engl J Med 2007;357(3):266-81.
18. Ho-Pham LT, Nguyen ND, Lai TQ et al. Vitamin D status and parathyroid hormone in a urban population in Vietnam. Osteoporos Int 2011;22(1):241-8.

19. Ho Chi Minh City Facts and Figures. www.hochiminhcity.gov.vn. Accessed July 10, 2008.

20. Kung AW, Lee KK. Knowledge of vitamin D and perceptions and attitudes toward sunlight among Chinese middle-aged and elderly women: A population survey in Hong Kong. BMC Public Health 2006;6:226.

21. R Development Core Team. R: A language and environment for statistical computing. 2.7.0. Vienna, Austria: $\mathrm{R}$ Foundation for Statistical Computing, 2008. http://www.R-project.org.

22. Bouillon R, Norman AW, Lips P. Vitamin D deficiency. N Engl J Med 2007;357(19):1980-1.

23. Lips P. Vitamin D physiology. Prog Biophys Mol Biol 2006;92(1):4-8.

24. Wat WZ, Leung JY, Tam S et al. Prevalence and impact of vitamin D insufficiency in southern Chinese adults. Ann Nutr Metab 2007;51(1):59-64.

25. Green TJ, Skeaff CM, Rockell JE et al. Vitamin D status and its association with parathyroid hormone concentrations in women of child-bearing age living in Jakarta and Kuala Lumpur. Eur J Clin Nutr 2008;62(3):373-8.

26. Hawkins RC. $25-\mathrm{OH}$ vitamin D3 concentrations in Chinese, Malays, and Indians. Clin Chem 2009;55(9):1749-51.

27. Holick MF. The vitamin D epidemic and its health consequences. J Nutr 2005;135(11):2739S-48S.

28. Stechschulte SA, Kirsner RS, Federman DG. Vitamin D: Bone and beyond, rationale and recommendations for supplementation. Am J Med 2009;122(9):793-802. 